\title{
MUSCLE AFFERENTS OF TRIGEMINAL MESENCE- PHALIC TRACT NUCLEUS AND MASTICATION IN CHRONIC MONKEYS
}

\author{
Ken'ichi Matsunami and Kisou Kubota \\ Department of Neurophysiology, Primate Research Institute, Kyoto University, \\ Inuyama City, Aichi, Japan, 484
}

\begin{abstract}
Summary The nature of masticatory movement and related muscle spindle afferent activity was studied on awake monkeys.

The pressure acting on the molar surface of the tooth and masseter EMG may develop in two phases, i.e. an early jaw movement and a late biting.

Out of 20 identified spindle afferents, 10 units in ten monkeys showed a rate increase during jaw-muscle closing and the remaining 10 units showed a decrease of the rate. Although there were two phases to the masseter contraction, no correlation was found between the two phases and the spindle afferent discharge pattern. It was concluded that during voluntary jaw closing the fusimotor activation occurred simultaneously with masseter muscle activation.
\end{abstract}

Research has shown that the fusimotor system is coactivated with the skeletomotor system when peripheral inputs or central structures are stimulated in the anesthetized and decerebrate states (GRAINT, 1970; DAVEY and TAYLOR, 1966; SEVERIN et al., 1967), and fusimotor activation often precedes the skeletomotor activation (gamma leading). This finding led to the idea that voluntary movement may be initiated by an initial fusimotor activation followed by reflexly evoked activation of skeletomotor neurons.

Stimulation of the primate motor cortex in the anesthetized state, however, does not induce the gamma leading that would be expected if the movement were initiated in the motor cortex, where the voluntary contraction is believed to be initiated by excitation (Koeze et al., 1968; LewIS and PorTer, 1971).

The purpose of this study is to determine whether in a voluntary movement the muscle spindle afferent activity attributable to the efferent fusimotor neuron activity may precede the activation of skeletomotor neurons. The trigeminal mesencephalic tract nucleus (TMT) was used as a target for recording single muscle spindle afferent in the unanesthetized chronic state. Experiments

Received for publication June 15, 1972

松波謙一, 久保田競 
were carried out on awake monkeys during voluntary jaw movements (a preliminary report was presented at the 47th annual meeting of the Japan Physiological Society in 1970).

\section{METHODS}

Seventeen adult macaque monkeys (11 Macaca mulatta and 6 Macaca irus) weighing $2.5-7.9 \mathrm{~kg}$ were used.

Operative procedures. Preliminary operations were performed under phencyclidine chloride anesthesia $(1 \mathrm{mg} / \mathrm{kg})$. The receptacle for the microdrive, a stimulating electrode for unit identification, a Kirschner wire, and a transducer to the tooth crown were implanted.

A hole about $20 \mathrm{~mm}$ in diameter and centered at $\mathrm{AP}=0, \mathrm{~L}=0$ (stereotaxic coordinates) was drilled in the vertex of the skull. A stainless steel cylinder 25-30 $\mathrm{mm}$ in length with an $18-\mathrm{mm}$ inner diameter was then attached to the bone and served as a receptacle for a hydraulic microdrive (EvARTS, 1968). Bolts and nuts were also implanted and used to fix the head rigidly during experiments (KUBOTA and NiKI, 1971). A conventional concentric stainless steel needle electrode $(0.6 \mathrm{~mm}$ outer diameter) was inserted through the brain into the semilunar ganglion ( $\mathrm{SLG}$; coordinates, $\mathrm{A}=7$ and $\mathrm{L}=11$ ) and was used to evoke a muscle twitch.

A Kirshner wire $(1.5 \mathrm{~mm}$ in diameter) was also inserted into the body of the mandible immediately dorsal to the mental foramena. Small loops at both ends of the wire were used for attaching weights (less than $500 \mathrm{~g}$ ) or strain-gauge transducers during the experiment.

In two monkeys a small strain gauge $(2 \times 2 \mathrm{~mm}$, p. 102, Shinkoh Tsushin) was inplanted in the first molar crown of the lower jaw. The dorsal surface of the molar was removed and flattened, the tooth pulp exposed, and the nerve killed by crushing. The gauge was covered with a thin layer of dental cement so that a force applied perpendicularly to the molar surface during biting could be detected.

Recording. Experiments were started as soon as monkeys recovered from the surgery, which took about one week. A monkey in the primate chair was restrained until the activity of TMT (trigeminal mesencephalic tract) neurons was recorded by means of polyurethane-coated tungsten electrodes (300 $\mu \mathrm{m}$ in diameter) with 2-10 M $\Omega$ resistances measured at $50 \mathrm{~Hz}$. The electrodes were attached to the hydraulic microdrive and inserted into the brain stem through the dura mater. As measured from the reading of the microdrive, the distance from the exposed dural surface to the TMT nucleus was about $18-22 \mathrm{~mm}$.

During experiments the EMG activity was recorded by a pair of bared thin copper wires from the masseter muscle ipsilateral to the side from which unit recording was done. Mechanical displacement of the jaw was recorded 
with a strain gauge (SB-1T, Nihon Kohden) and a strain meter (DS6/MTC, Shinkoh Tsushin). The gauge was hooked to the end loop of the Kirshner wire. An accelerometer (Type BA2-120, Shinkoh Tsushin) was also attached to the end loop and its output fed to the strain meter. All parameters were displayed on a cathode-ray oscilloscope (VC-7, Nihon Kohden) and two of them were also recorded on a 2-channel tape recorder (TEAC 4010S).

Identification of units. In order to identify a given unit to be of muscle spindle origin from the TMT nucleus, a brief rectangular pulse $(0.1 \mathrm{msec}$ duration, 1.5-5V, Type MSE-3 electronic stimulator, Nihon Kohden) was applied through the electrode in the SLG. When, during muscle twitch, a unit showed a period of inactivity in phase with the contraction period, that unit was considered to be a TMT spindle afferent (see JERGE, 1963).

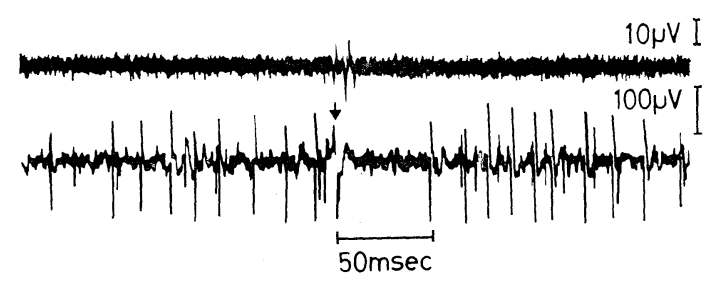

Fig. 1. Identification of a single unit in the trigeminal mesencephalic tract nucleus (TMT) as a muscle spindle afferent. The semilunar ganglion (SLG) was stimulated by a brief single pulse at the moment indicated by the arrow. Upper trace: masseter muscle EMG. Lower trace: single unit activity recorded from the TMT nucleus. Note the absence of activity for $50 \mathrm{msec}$ after a shock.

Figure 1 shows an example of a pause in single unit activity when the jawclosing muscle was contracted by a pulse applied to the SLG. After a pulse a small fraction of the evoked EMG was detected. In this example the pause in activity of the TMT unit was about $50 \mathrm{msec}$.

In total, 20 units were identified as originating from muscle spindles. The duration of the pause varied from unit to unit and depended on the state of the muscle contraction, whether it was shortened or relaxed, or whether an afferent had the so-called gamma bias or not. Since contraction time of the jaw-closing muscle was short (16 msec, see HARrISON, and CORBIN 1942), it was difficult to confirm the presence of a pause during twitch, when the discharge rate was low, or the mouth closed. Therefore, testing was most conveniently done when the mouth was opened by passive stretching. The pause fell between 30 and 140 msec, the mean value of 20 units being $63 \mathrm{msec}$. Firing rate of identified units increased if pressure was applied to the masseter, temporal, or pterygoid muscles. Exact location of the spindle was therefore difficult to determine in all units.

Conduction velocity was not measured for any of the units. At the end of recording, $\mathrm{DC}$ current $(5 \mu \mathrm{A}$ for $10 \mathrm{sec})$ was passed through the recording 
electrode and a small lesion was made for later histological identification of the recording point. At the end of the experiments the monkey was sacrificed, Ringer's solution was perfused from the carotid arteries, and the brain was fixed with formalin. The brain was cut serially at $50 \mu$ thickness and stained with cresyl violet.

Out of the 20 identified units, 14 points were ascertained histologically, as illustrated by the filled circles in Fig. 2. Most of these fell in planes between $\mathrm{P}=0$ and $\mathrm{P}=2$. Laterally they were between $\mathrm{L}=0$ and $\mathrm{L}=2$.

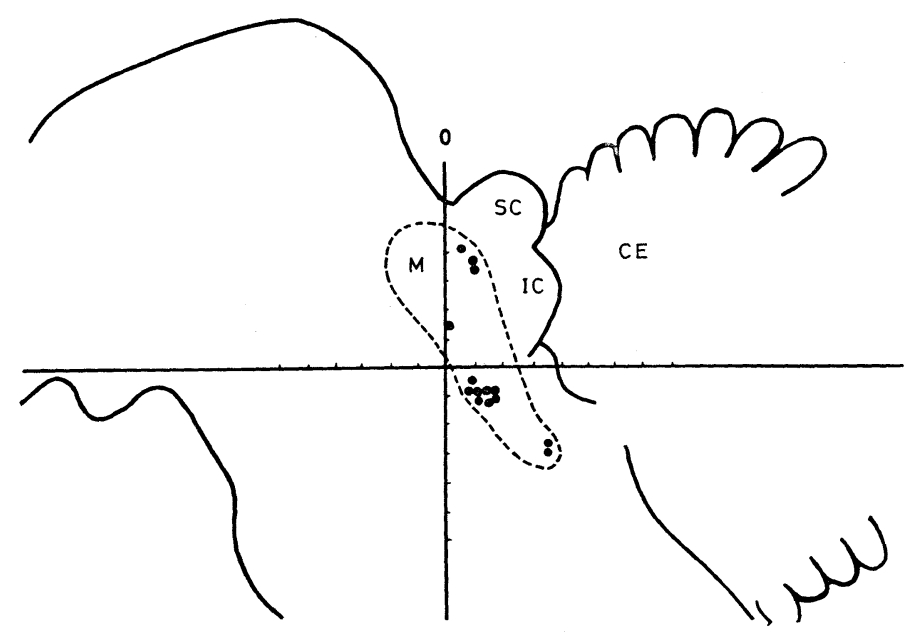

Fig. 2. Anatomical locations of functionally identified muscle spindle afferent units. The location of the units were projected on the midline sagittal plane, which was reconstructed from SNIDER and LeE's atlas (SNIDER and LEE, 1961). All units are included in the TMT nucleus. The nucleus location projected on the sagittal plane is enclosed by a broken line. $\mathrm{M}$ : mesencephalic tract nucleus. SC: superior colliculus. IC: inferior colliculus. $\mathrm{CE}$ : cerebellum. Zero indicates $\mathrm{AP}=0$ level.

\section{RESULTS}

Results will be described in two sections. In the first, the relation between the EMG activity of the masseter muscle and induced changes of the mechanical displacement or of the muscle force will be described. The presence of two phases in the development of the jaw-closing muscle activity will be dealt with. Second, discharges of muscle spindle afferents during mastication will be compared with the EMG activity.

\section{Two phases of biting}

The masseter EMG activity during mastication was often characterized by a gradual increase during the early phase followed by a rapid increase to maximum 
amplitude near the end of contraction. Thereafter the EMG activity terminated abruptly (see Figs. 3B and 4C). This observation suggested that the biting (jawclosing muscle contraction) might develop in two phases: in the early phase the lower jaw is elevated toward the upper jaw, and in the late phase contact of the teeth is accomplished by a recruitment of motor units producing the force for the actual biting.

The force applied to the surface of a molar tooth of the lower jaw and the EMG from the masseter were simultaneously recorded in a single episode of jaw closing and are illustrated in Fig. 3. Phases similar to those just described for the EMG were also observed in the record of force applied to the molar surface. During the early phase force slowly increased and during the late phase it abruptly increased to its maximum value. The two phases corresponded closely

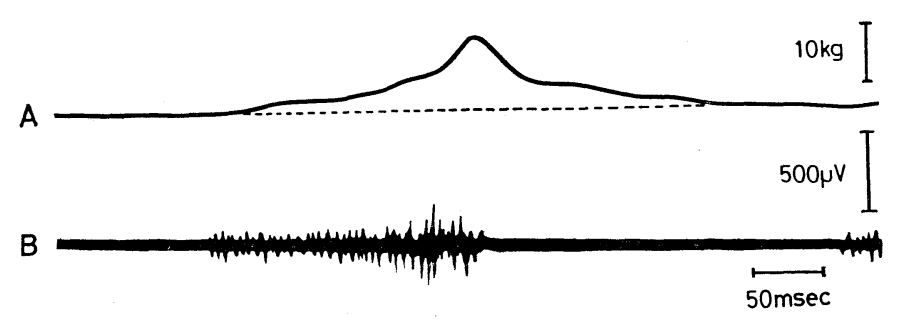

Fig. 3. Relation between the pressure change imposed on the surface of the first molar tooth

(A) and the masseter EMG (B). Time and sensitivities are indicated at right.

in time with the phases of the EMG activity. Following the maximum level of activity the EMG returned rapidly to the initial level, while the pressure returned gradually. This slow return of pressure is not well explained. In tested monkeys two phases in the development of force by the masseter to the molar tooth were observed in about $50 \%$ of the trials of jaw closing. In the other trials the two phases were not clearly separated. Whenever the two phases appeared they were observed for several successive trials. The reason for this was not analyzed but may be related to the fact that the state of the food in the mouth changes with repeated bitings.

The jaw bone acceleration, its displacement, and the masseter EMG were simultaneously recorded in two monkeys and interrelations among these three indices were studied. An example of these curves during successive episodes of biting are illustrated in Fig. 4, in which the top trace (A) shows the displacement as measured by a strain gauge attached to the lower jaw, the middle (B) shows the acceleration as measured by an accelerometer attached to the lower jaw, and the bottom trace $(\mathrm{C})$ shows the EMG recorded from the masseter muscle. An upward displacement of the jaw in (A) started slightly after the onset of the EMG in (C). As seen in curve A, displacement developed in two stages. Ini- 
tially, from point 2 to point 3, the lower jaw moved upward. Thereafter, the position remained nearly constant, although the EMG activity continued. The EMG after point 3 is larger in size than before that point. As for the acceleration curve (B), the steepest rise was seen in the earliest phase of the EMG, from point 3 to 4 . It reached a peak value at point 4, $25 \mathrm{msec}$ after the EMG onset, and then decreased gradually, which was about $100 \mathrm{msec}$ earlier than the end of the EMG activity.

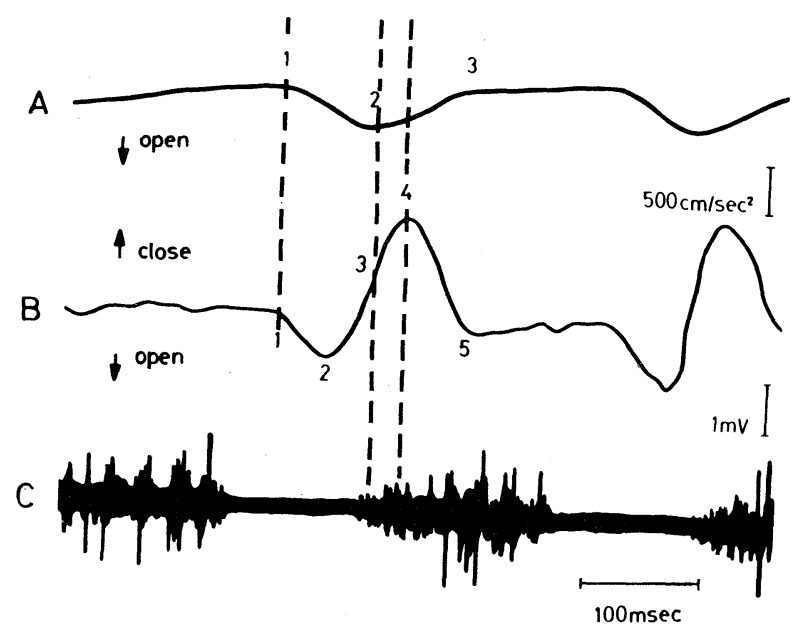

Fig. 4. Simultaneous recordings of jaw movement (A), acceleration (B), and masseter EMG (C). Three vertical broken lines were drawn to indicate time relations more clearly. In A, point 1 denotes the beginning of mouth opening, point 2 maximum mouth opening, and point 3 closing of the mouth. In $\mathrm{B}$, point 1 shows the beginning of acceleration for the opening, point 2 indicates the timing of maximum acceleration for opening, and point 3 indicates the beginning of the closing movement. Note that the masseter EMG continues after the end of the mechanical displacement.

A downward displacement (jaw opening) started $40 \mathrm{msec}$ after the end of the masseter EMG (point 1 of $\mathrm{A}$ and $\mathrm{B}$ ) and reached a maximum just before the EMG onset. Acceleration for the opening, starting simultaneously with the displacement, reached a peak (point 2, B) about 25 msec earlier than EMG onset. It is clear from Fig. 4 that the timing of the largest acceleration in the biting occurred at the early phase of the contraction. In two monkeys examined, the acceleration peak occurred 20-30 msec after the EMG onset, and maximum acceleration values for jaw closing were as large as $1.5 \mathrm{G}$.

From these data, it may be said that the early phase of the masseter EMG reflects mainly the jaw movement which brings the lower jaw closer to the upper jaw with the largest acceleration so that the teeth meet, and that the late phase reflects actual biting with large applied force. 


\section{Muscle afferents and mastication}

In the second part of the investigation, we attempted to see whether fusimotor activity may start earlier than skeletomotor activity in the initiation of voluntary movement. Afferents, identified as of muscle spindle origin, invariably had a spontaneous activity, the rate being always unsteady and falling in the range of $14-40 \mathrm{~Hz}$. The firing rate decreased or increased even when the animal sat quietly without any biting activity. The discharge pattern during biting was classified into three categories. Examples of these units are illustrated in Fig. 5.

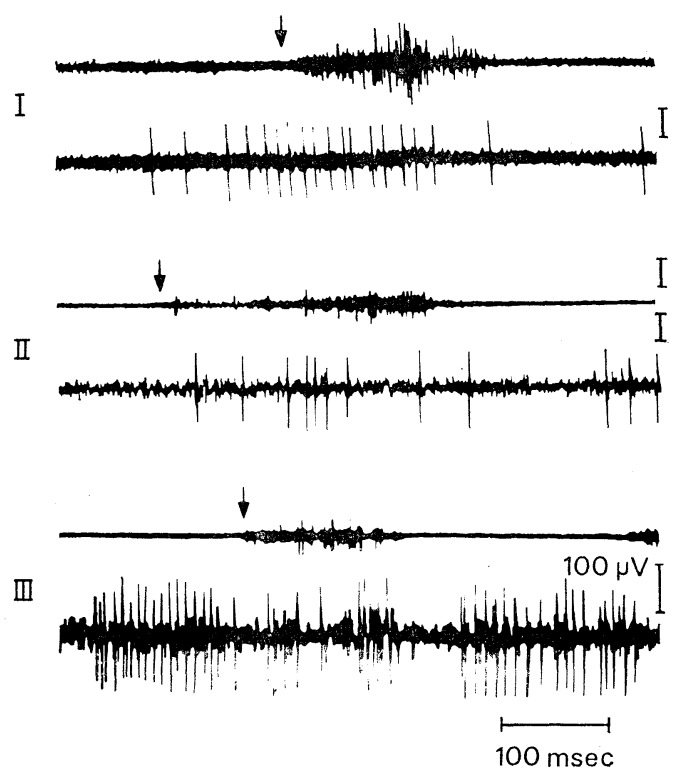

Fig. 5. Muscle spindle afferent discharge and masseter EMG. Three different patterns are illustrated in I, II, and III. Upper trace: masseter EMG. Lower trace: spindle afferent unit. Arrows indicate the EMG onset.

The unit in I shows an increased discharge rate about $80 \mathrm{msec}$ prior to the EMG onset. The discharge pattern continued to show an increased rate as long as EMG activity was present. The highest firing rate was at the onset period of the EMG. The end of EMG activity was followed by a silent period in the afferent. The unit illustrated in II of Fig. 5 began to show an increase in the firing rate slightly after the EMG onset and was active as long as the EMG was present. A silent period of about $120 \mathrm{msec}$ was also observed after the end of the EMG. In contrast to these two units, the unit illustrated in III showed less activity during biting and more activity when the EMG from the masseter was silent. A complete cessation of activity lasting for $70 \mathrm{msec}$ was present after the end of the 
EMG activity. Each identified unit showed one and only one of these three patterns.

Out of 20 identified units, 5 units showed the pattern seen in I, another 5 that seen in II, and the remaining 10 units showed firing patterns similar to III. In all units, however, there was a cessation of afferent activity for about $100 \mathrm{msec}$ after the end of the EMG activity. As for the third pattern, shown in III, depression usually started as soon as the EMG started, though in most cases it was observed only in the initial phase of the EMG. In the later phase unit activity returned to the previous rate during the jaw-opening phase. The scattering of the timing of an activity pattern like Fig. 5, I, is illustrated in Fig. 6. Zero (indicated with a vertical line) was adjusted to the onset time of the masseter EMG. In each unit ten trials of jaw-closing are represented. Each dot represents single spike discharge. Increase in firing rate was observed about $100 \mathrm{msec}$ preceding the EMG onset.

I

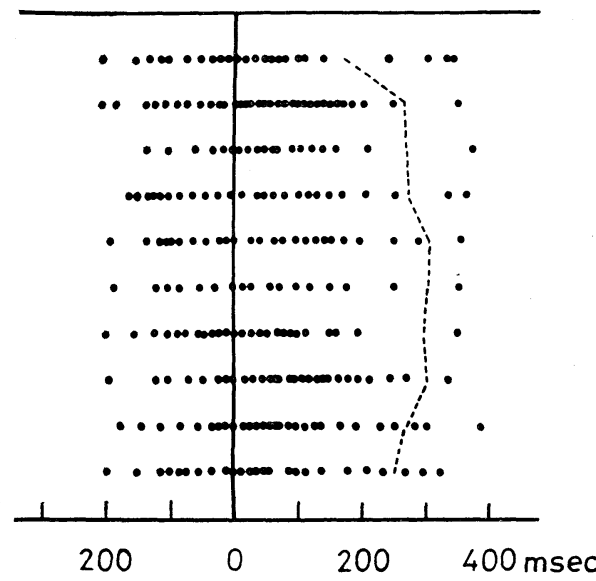

II

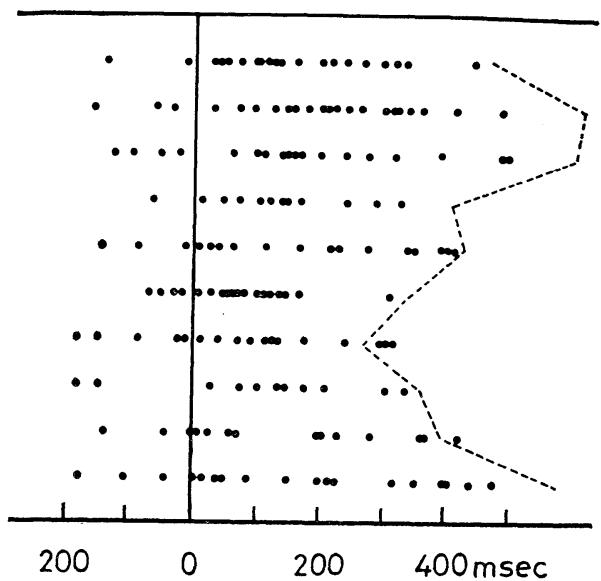

Fig. 6. Dot displays of two muscle spindle units during jaw closing. Each dot represents a spike discharge. A vertical line at zero represents the timing of EMG onset. Ten successive episodes of closing are illustrated in vertical order. The broken line signifies the end of EMG.

As for units active in the phase immediately preceding the start of EMG activity, as seen in Figs. 5 and 6, it was difficult to infer the timing of the fusimotor activation for voluntary jaw closing since the muscle spindle afferents may also be activated by the passive stretching of the masseter muscle induced by voluntary jaw opening. In order to observe any abrupt increase of the rate of the phase preceding EMG activity which may possibly be free from the change induced by 
passive stretch and may be due to the fusimotor activation, average responses in a series of succeeding trials were calculated. Spike numbers at 50-msec intervals of 6 different units were summed and plotted and are illustrated in the graphs in Fig. 7. In the graphs thus made, an abrupt increase of the rate is not seen
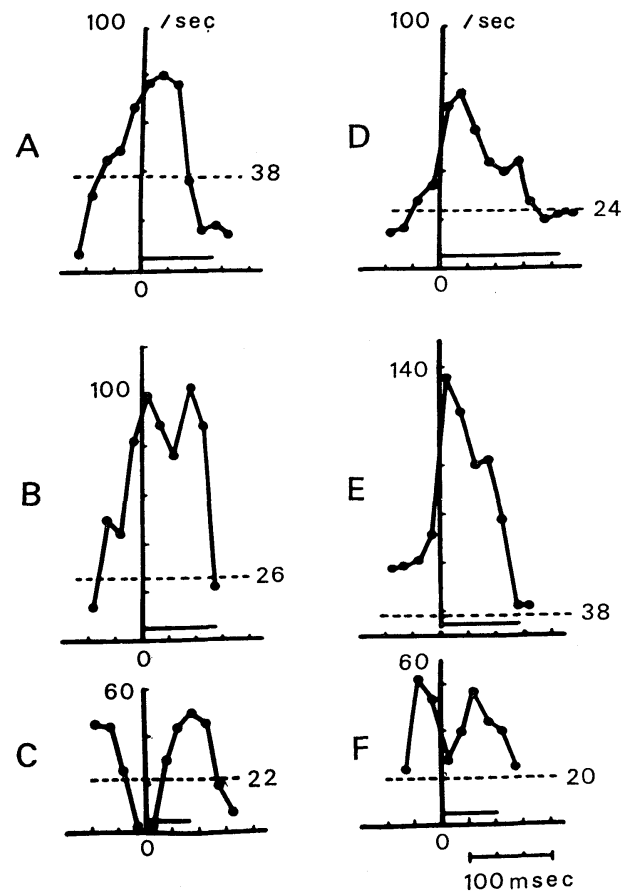

Fig. 7. Development of unit discharge in relation to time. A to F, 6 different units. Ordinate, average frequencies of 50-msec intervals. Abscissa, time. Horizontal dotted lines, average spontaneous frequencies. Horizontal bars, average duration of EMG.

in $\mathrm{A}$ and $\mathrm{B}$. In $\mathrm{D}$ an increase is seen simultaneous with the EMG onset, and in $E$ a slight increase is seen $50 \mathrm{msec}$ earlier than the EMG onset. In units $\mathrm{A}$ and $\mathrm{B}$, maximum activity is seen either in the early or middle phase of the masseter EMG. It is inferred in the cases of $\mathrm{D}$ and $\mathrm{E}$ that fusimotor activation started almost simultaneously with the EMG activation. In the responses shown in $\mathrm{C}$ and $\mathrm{F}$, a decrease is seen immediately preceding the onset of and during the early phase of EMG. Thus, in these two the fusimotor activation is not powerful enough to overcome the decrease of the rate by muscle contraction. A clear, abrupt, discontinuous increase of the rate prior to the EMG onset was not seen in any of the units tested. 


\section{DISCUSSION}

Muscle spindle activity of the mesencephalic tract nucleus innervating the jaw-closing muscle, presumably the masseter muscle, was compared with the masseter EMG and actual displacement of the jaw. In the early phase of the masseter contraction the largest acceleration and the displacement of the jaw were observed. In the late phase no significant acceleration on displacement was observed, but the masseter EMG activity was more striking and persistent and the biting tension was stronger. It may be said, therefore, that the muscle activity in the late phase is for biting without appreciable isometric contraction. Although separation of the masseter activity into two phases is observable, no clear separation of the discharge pattern of muscle spindle afferents was observed. In half of the identified spindle afferents fusimotor activation was seen to occur almost simultaneously with the masseter EMG. In no units was there a discharge increase which would indicate gamma leading. It can be said, therefore, that the fusimotor activation occurred simultaneously with the masseter activation. The spindle activation was observable as long as the muscle was contracting, whether it was in the initial isotonic or the late isometric phase. These results are consistent with the finding that there is no gamma leading produced by motor cortex stimulation in the primate (KoEze et al., 1968).

In about half of identified units depression was observed during jaw closing. In these units it is not clear whether the depression is due simply to a reflection of the fact that fusimotor innervation may have been less in these units or is due to an inherent technical artifact. In the operative procedure parts of the temporal muscle fibers were partially removed from the skull so that the cylinder could be implanted. This procedure may have caused degeneration of fibers of the temporal muscles, including intrafusal fibers, possibly producing the decreased activity of the spindle afferents. Since the origin of the afferents was not clearly determined, these depressed units may have arisen from terminals belonging to degenerated fibers. Cessation of the discharge was not clearly explained but may be due to the viscoelasticity of the intrafusal muscle fibers. It is also possible that an inhibitory mechanism is activated at the fusimotor neuron level. By contact of, for example, upper and lower teeth the inhibitory afferents from the periphery may depress the jaw-closing motoneurons (both alpha and gamma).

The authors are grateful to Miss Takako Honda for her assistance on histology.

\section{REFERENCES}

Davey, M. R. and TAYlor A. (1966) Activity of cat jaw muscle stretch receptors recorded from their cell bodies in the mid-brain during spontaneous jaw movements. J. Physiol. (London), 185: 62-63.

EVARTS, E. V. (1968) A technique for recording activity of subcortical neurons in moving animals. Electroencephalogr. Clin. Neurophysiol., 24: 83-86. 
Graint, R. (1970) The Basis of Motor Control. Academic Press, London, p. 346.

Harrison, F. and Corbin, K. B. (1942) The central pathway for the jaw-jerk. Am. J. Physiol., 135: 439-445.

JERGE, C. R. (1963) Organization and function of the trigeminal mesencephalic nucleus. $J$. Neurophysiol., 26: 379-392.

Koeze, T. H., Phillips, C. G., and Sheridan, J. D. (1968) Thresholds of cortical activation of muscle spindles and alpha-motoneurones of the baboon's hand. J. Physiol. (London), 195: 419-449.

KubotA, K. and Niki, H. (1971) Prefrontal cortical unit activity and delayed alternation performance in monkeys. J. Neurophysiol., 34: 337-347.

Lewis, M. MCD and PorTer, R. (1971) Lack of involvement of fusimotor activation in movements of the foot produced by electrical stimulation of monkey cerebral cortex. J. Physiol. (London), 212: 707-718.

Severin, F. V., OrlovskiI, G. N., and ShiK, M. L. (1967) Work of the muscle receptors during controlled locomotion. Biophysics, 12: 575-586 (Eng. trans. of Biofizika, 12: 502-511).

Snider, R. S. and LeE, J. C. (1961) A Stereotaxic Atlas of the Monkey Brain (Macaca mulatta). The Univ. of Chicago Press, Chicago. 